\title{
Sugar Cane (Saccharum officinarum L.) Analysis through Biospeckle and Spectroscopy (NIR)
}

\author{
Matheus Sevillano Marcondes ${ }^{1}$, Keylla Guiguer Silva ${ }^{1}$, Armando Kazuo Fujii ${ }^{1}$, Ronei Jesus Poppi ${ }^{2}$ and Juliana \\ Aparecida Fracarolli ${ }^{1}$ \\ 1. Department of Pre-processing Agricultural Products, School of Agricultural Engineering, University of Campinas, Campinas, São \\ Paulo 13083875, Brazil
}

2. Department of Analytical Chemistry, Institute of Chemistry, University of Campinas, Campinas, São Paulo 13083970, Brazil

\begin{abstract}
The objective of this paper was to evaluate the sugar and water content in sugar cane samples by means of two non-destructive techniques in comparison with conventional methods. One non-destructive technique is the Biospeckle, an interaction between the laser and the biological material which provides information about the material. The other technique is the near infrared spectroscopy (NIR). The Biospeckle technique has been constantly used in agricultural engineering. In this paper, the relation between the water and the sugar contents were obtained. The results for the Biospeckle were acquired through the moment of inertia (MI). The data acquired through already consolidated methodologies were used to quantify the samples' water and sugar contents, correlating to the spectroscopy results and also to the Biospeckle. It was obtained a high determination coefficient $\left(R^{2}=\right.$ 0.9287) between the predicted Brix by the model generated by NIR spectroscopy and Brix measured by refractometer. It was also possible to differentiate the water contents of the sugar cane samples both externally lit (husk) and internally lit (cut) by Biospeckle analysis through the calculation of MI. It is possible to evaluate the water content and the sugar content in samples combining Biospeckle and NIR spectroscopy, which are non-invasive and non-destructive methods.
\end{abstract}

Key words: Sugar content, water content, non-destructive analysis, dynamic speckle, image processing, laser.

\section{Introduction}

Currently, in order to determine if the sugar cane is at maturation point for harvesting process, a field refractometer is used. It is a device which provides the percentage of soluble solids of the broth, which is linked to the sugar cane sucrose content. After that measurement, a laboratorial analysis is performed. The knowledge on the water content of the samples is also important for ethanol and sugar productivity estimates. So, in the harvest automation of agricultural products, researches for non-destructive analyses of biological materials have been developed.

In that context, the near infrared spectroscopy (NIR), an electromagnetic interval, has been used in studies related to sugar cane, as proposed by Lemões et al. [1], in which the NIR technique is used on the

Corresponding author: Juliana Aparecida Fracarolli, assistant professor, research field: biospeckle. construction of spectral curves and thus makes it possible to predict the total reducing sugar and sugar values. The NIR is an analytical technique, which allows dosage of several matter constituents in a fast and non-destructive way, and has often been applied in an advantageous way to determine organic molecules and qualitative parameters of food and agricultural products [2].

Nawi et al. [3] identified that the sugar content varied along the position of the sugar cane stem. Ferraz [4] obtained reading results performed directly from the culms on the outer surface, and presented correlations between the spectroscopy and Brix, making it possible to perform an analysis of the sugar cane in a fast and efficient way, without destruction of the sample.

On the other hand, the Biospeckle technique has been widely used on the evaluation of fruit maturity, 
seed feasibility, bovine semen mobility, hydric deficit in plants, and other applications [5-7]. It presents advantages, since it is non-destructive and non-invasive. It also permits to automate the evaluation of biological materials. One of the ways to obtain results through Biospeckle is by calculating the moment of inertia (MI). It is also possible to correlate the moisture content of the biological material with the MI [8].

This research aimed to analyze the water and sugar content in sugar cane samples, through two non-destructive techniques—Biospeckle and NIR.

\section{Materials and Methods}

The Biospeckle tests were conducted in the Optics Laboratory and in the Post-Harvest Laboratory of the School of Agricultural Engineering (FEAGRI/UNICAMP). The spectroscopy of the samples was performed in the Laboratory of the Analytical Chemistry Automation and Instrumentation Group of the Chemistry Institute (IQ/UNICAMP).

The equipment used for the Biospeckle was: light source with diode laser with a wavelength of $632 \mathrm{~nm}$ and $3 \mathrm{~mW}$ of power, digital camera, plane mirror and computer with a core i7 processor.

The MI is calculated through the speckle spectral variation. In order to obtain the values for study, the time history speckle pattern (THSP) is used, obtaining the values through the manipulation of several images acquired from the surface. Those images are captured at time intervals in the order of milliseconds. For each acquired image, a sample column is extracted from the center. With that, it is possible to obtain a sequence, where the height corresponds to the column size in pixels and the width corresponds to the number of captured images. The MI is calculated as Eq. (1):

$$
\mathrm{MI}=\sum_{i j} M_{i j}(i-j)^{2}
$$

where, MI corresponds to the moment of inertia values; indexes $i$ and $j$ are the respective positions in the occurrence matrix which also correspond to the intensities. While, $M_{i j}$ is obtained through Eq. (2):

$$
M_{i j}=\frac{N_{i j}}{\sum N_{i j}}
$$

where, $N_{i j}$ corresponds to the number of occurrences of intensities.

The equipment used for the NIR spectroscopy was: tungsten halogen lamp, Fourier transform infrared (FTIR) spectrometer in the range of 4,000 wavenumbers $/ \mathrm{cm}$ to 10,000 wavenumbers $/ \mathrm{cm}$ and deuterated-triglycine sulfate (DTGS) detector.

Fifteen plants of sugar cane variety RB867515 were collected in the field at the fifth cut stage from May 2011 planting.

The initial water content was obtained in a kiln at $130^{\circ} \mathrm{C}$ for $2 \mathrm{~h}$. The total soluble solids (TSS) content was also obtained in refractometer (ATAGO N1).

\subsection{Water Content}

In order to evaluate the samples' water content, first the culms of each plant at the lower part near the root, were cut in equal sections, summing 48 repetitions for two treatments- $62 \%$ and $71 \%$ wet basis of moisture content. The samples were placed in a kiln at $70{ }^{\circ} \mathrm{C}$ for $1 \mathrm{~h}$ in order to obtain the moisture variation.

After determining the initial moisture content of the samples, the amount of dry matter was determined. After the dry matter quantity was reached, all the repetitions were weighed to determine the moisture of each repetition.

The analysis of water content and Biospeckle were performed in the same day and with the same samples. For biospeckle analysis, the samples were illuminated in sequence and the samples were analyzed in the oven to obtain the moisture content. The samples were hermetically conditioned so that there were no alterations in sugar and water content levels.

For the analysis of the samples, 20 seconds videos were made with a camera. Each sample was internally and externally (over the husk) illuminated with the laser to obtain the MI. Then, the MI obtained from the 
sugarcane (internally and over the husk) was statistically related to the water content of the sample. The procedure aims to a future procedure for determining the moisture in a non-destructive way.

The MI values were obtained for each repetition in the different moisture content. The data were analyzed through Tukey’s test at 5\% probability.

\subsection{Sugar Content}

For each plant, the culms were cut in three equal sections (low part, medium part and high part of the culm) and divided in husk and cut. There were 15 repetitions for each analysis of TSS in the refractometer, NIR (husk and cut) and Biospeckle (husk and cut), according to Table 1.

For the initial analysis, the Brix results was obtained from the broth by the spectral readings. 10 principal components were used in the model development in order to analyze the data obtained from NIR (principal component analysis). Then, the spectral readings calibration was performed. A pre-treatment of the data was performed, using the multiplicative scatter correction (MSC) for baseline correction. This transformation is used to correct additive and multiplicative effects on absorbance caused by physical phenomena that have no relation to the composition of the analyzed sample [9].

The average Brix results in the different sections inside the same plant were analyzed through Tukey's test at $5 \%$ probability and compared to the spectral readings, through multivariate analyses and partial least-square regression (PLSR) techniques.

\section{Results and Discussion}

\subsection{Water Content}

Total 96 plants were lighted repesctively from external part (husk) and internal part (cut), with 48 plants for each treatment. And then Biospeckle analysis was used by calculation of MI values to distinguish the different moisture. As shown in Table 2, the coefficient of variation (CV) was $57.09 \%$ for the externally lit samples, while for samples lit on the cut (internal part), CV of MI was 75.14\%.

From Table 2, it is possible to observe the differentiation in the water contents of the samples lit from the husk and cut. From these results, it can be inferred that it is possible to analyze the samples moisture without destroying or cutting the samples.

\subsection{Sugar Content}

Total 45 plants were analyzed with 15 repetitions respectively for apex, middle and base part of culms. For the analysis among culms of the apex, central and base part of the sugar cane, the results for samples lit on the husk (external part) and the central part (internal part) were shown on Table 3. If lit on the

Table 1 Number of samples and repetitions.

\begin{tabular}{llllll}
\hline \multirow{2}{*}{ Section of samples } & \multicolumn{5}{c}{ Repetitions for each analysis } \\
\cline { 2 - 6 } & Refractometer & NIR (husk) & NIR (cut) & Biospeckle (husk) & Biospeckle (cut) \\
\hline Low part of culm & 15 & 15 & 15 & 15 & 15 \\
Medium part of culm & 15 & 15 & 15 & 15 & 15 \\
High part of culm & 15 & 15 & 15 & 15 & 15 \\
\hline
\end{tabular}

Table 2 MI values of the externally and internally lit samples by Biospeckle analysis at different water contents.

\begin{tabular}{lll}
\hline \multirow{2}{*}{ Water content } & \multicolumn{1}{c}{ MI averages } \\
\cline { 2 - 3 } & Externally lit samples (husk) & Internally lit samples (cut) \\
\hline $72 \%$ & $1.196954^{\mathrm{a}}$ & $1.157921^{\mathrm{a}}$ \\
$61 \%$ & $1.844317^{\mathrm{b}}$ & $3.496188^{\mathrm{b}}$ \\
\hline $\mathrm{CV}$ & $57.09 \%$ & $75.14 \%$ \\
\hline
\end{tabular}

Averages followed by the same letter do not statistically differ through Tukey’s test $(P<0.05)$. 

and Spectroscopy (NIR)

Table 3 MI values obtained for the different part of culms (the apex, the central and the base) by Biospeckle analysis.

\begin{tabular}{lll}
\hline \multirow{2}{*}{ Part of culms } & \multicolumn{1}{c}{ MI averages } \\
\cline { 2 - 3 } & Externally lit (on the husk) & Internally lit (on the cut) \\
\hline Apex & $3.203773^{\mathrm{a}}$ & $3.847407^{\mathrm{b}}$ \\
Middle & $1.581753^{\mathrm{b}}$ & $3.810333^{\mathrm{b}}$ \\
Base & $1.155380^{\mathrm{b}}$ & $2.418773^{\mathrm{b}}$ \\
\hline CV & $38.53 \%$ & $60.87 \%$ \\
\hline
\end{tabular}

Averages followed by the same letter do not statistically differ through Tukey’s test $(P<0.05)$.

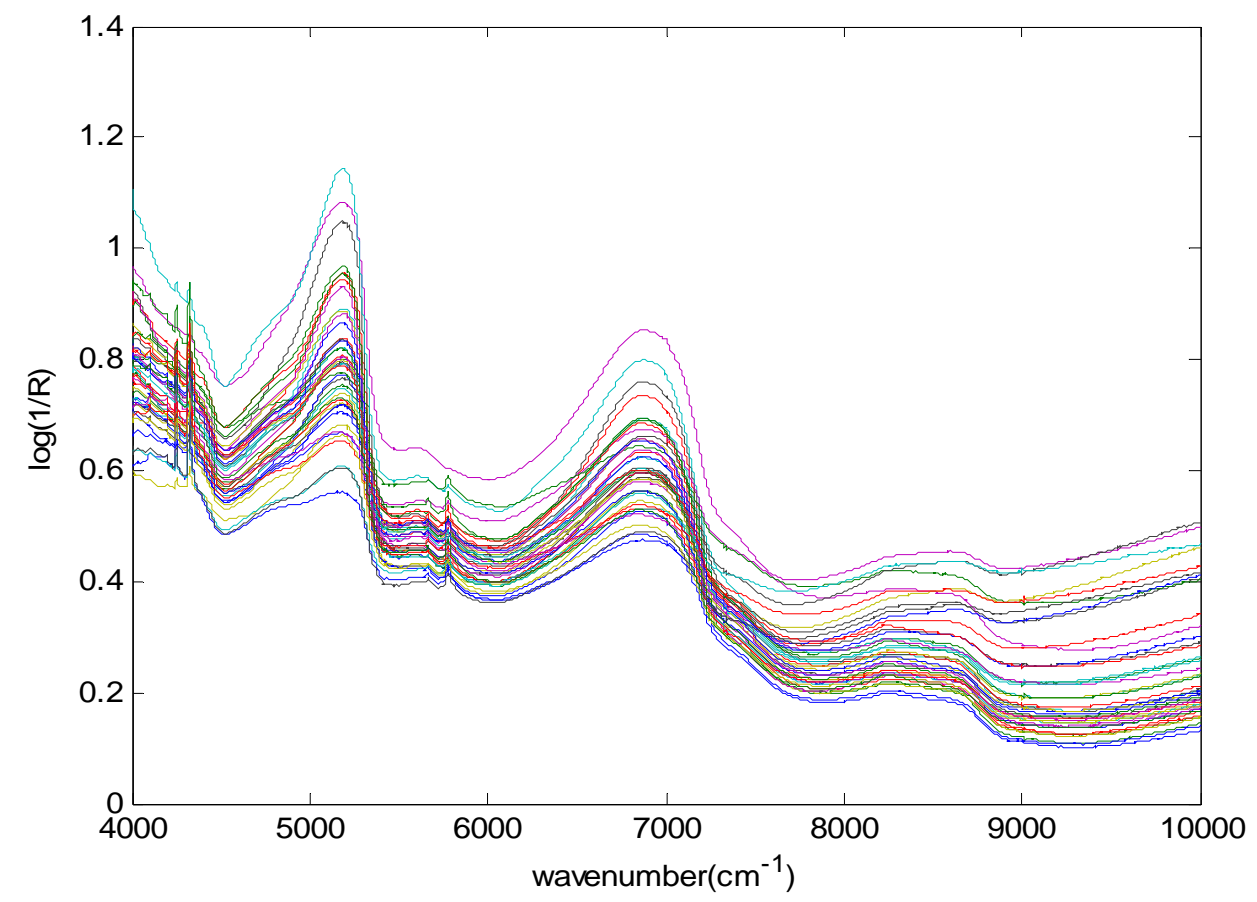

Fig. 1 Spectrum of the samples obtained from the husk by NIR.

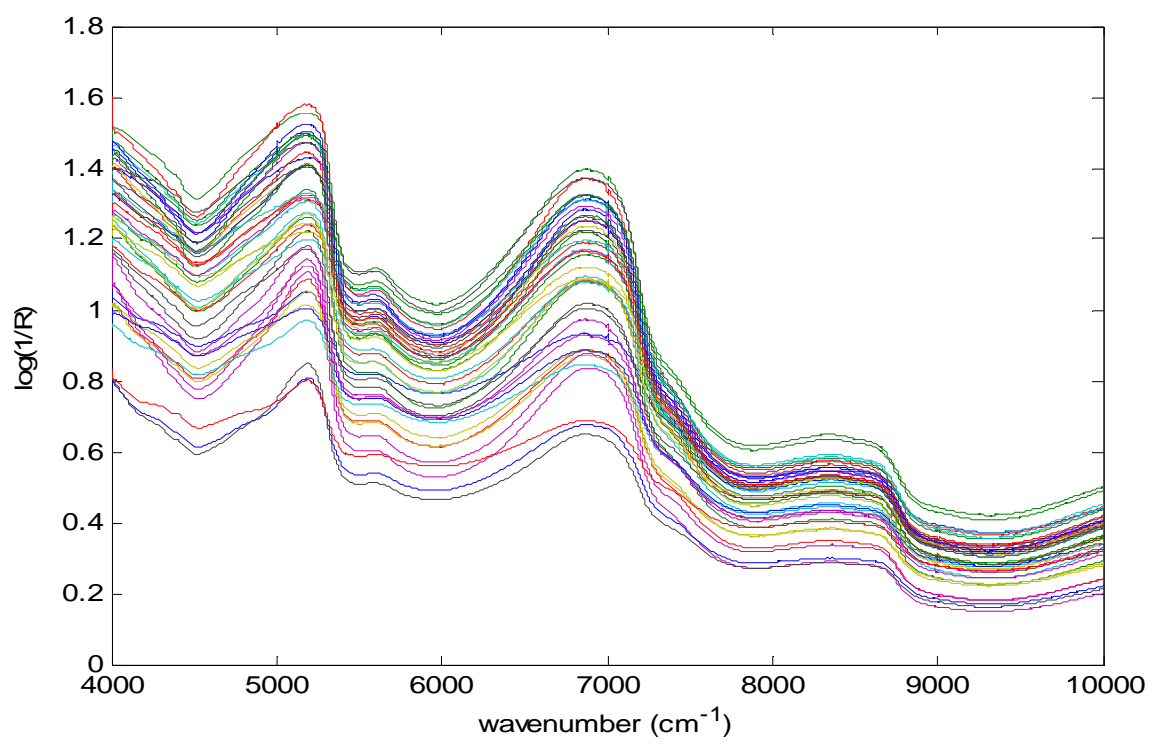

Fig. 2 Spectrum of the samples obtained from the internal part by NIR. 
husk, the $\mathrm{CV}$ was $38.53 \%$, while on the cut, CV was $60.87 \%$.

It is possible to observe that the MI was higher for the apex of the plant, in which area the plant presents high perspiration. And in this region, the growth of the plant also occurs, with the development of new cells. It is also a region which presents lower sugar content, but higher water content.

The spectrum of the samples obtained from the husk by NIR can be observed from Fig. 1, while the spectrum of the samples from the internal part of the samples by NIR can be observed from Fig. 2.

It can be seen that the treatments were not distinguished through Tukey's test at 5\% probability $(\mathrm{CV}=60.87 \%)$, when the images were acquired from the internal part of the samples for the Biospeckle (Table 3). However, the results for Biospeckle from the husk could be differentiated through Tukey's test $(\mathrm{CV}=38.53 \%)$. It is possible to observe that the samples lit on the husk present a statistical differentiation (Table 3), while the samples lit on the internal part were not statistically distinguished.

The sugar contents obtained through refractometer for the apex, center and base parts of the sugar cane were also subjected to Tukey's test at 5\% probability (Table 4). The coefficient of variation was $8.99 \%$ for three treatments ( $n=15$ for each treatment).

\subsection{Comparison of Brix Values Obtained through NIR and Refractometer}

The prediction of the Brix values obtained through NIR was compared with to the Brix values obtained from refractometer. Table 5 shows the results of the sugar content ( ${ }^{\circ}$ Brix) predicted through NIR, in correlation to the sugar content values obtained in refractometer, with MSC pre-processing and PSLR method, for samples obtained from the husk and the internal part, respectively.

Table 4 The sugar content for culms obtained from the apex, middle and base of the plant through a refractometer.

\begin{tabular}{ll}
\hline Parts of culms & Sugar content $\left({ }^{\circ}\right.$ Brix $)$ \\
\hline Apex & $8.332667^{\mathrm{a}}$ \\
Middle & $18.184000^{\mathrm{b}}$ \\
Base & $20.284667^{\mathrm{c}}$ \\
\hline CV & $8.99 \%$ \\
\hline
\end{tabular}

Averages followed by the same letter do not statistically differ through Tukey's test $(P<0.05)$.

Table 5 Prediction of the sugar content of the samples for model validation .

\begin{tabular}{lll}
\hline Measured value by the refractometer & Predicted NIR value (husk) & $\begin{array}{l}\text { Predicted NIR value } \\
\text { (internal part) }\end{array}$ \\
\hline 8.7000 & 11.0353 & 10.6528 \\
10.7900 & 9.1393 & 9.5448 \\
7.3800 & 7.3662 & 8.2518 \\
7.8500 & 7.1695 & 7.9900 \\
7.8000 & 9.5305 & 8.0395 \\
19.7100 & 18.7624 & 18.1236 \\
18.6100 & 18.3029 & 19.5586 \\
20.9500 & 16.1805 & 19.7173 \\
19.1300 & 19.0043 & 17.0032 \\
19.2200 & 18.8743 & 17.7823 \\
18.0200 & 17.7249 & 16.0269 \\
16.1900 & 18.8010 & 17.4355 \\
16.4800 & 17.9421 & 17.6875 \\
18.1600 & 15.0683 & 19.7603 \\
20.9500 & 17.5003 & 19.7862 \\
\hline
\end{tabular}




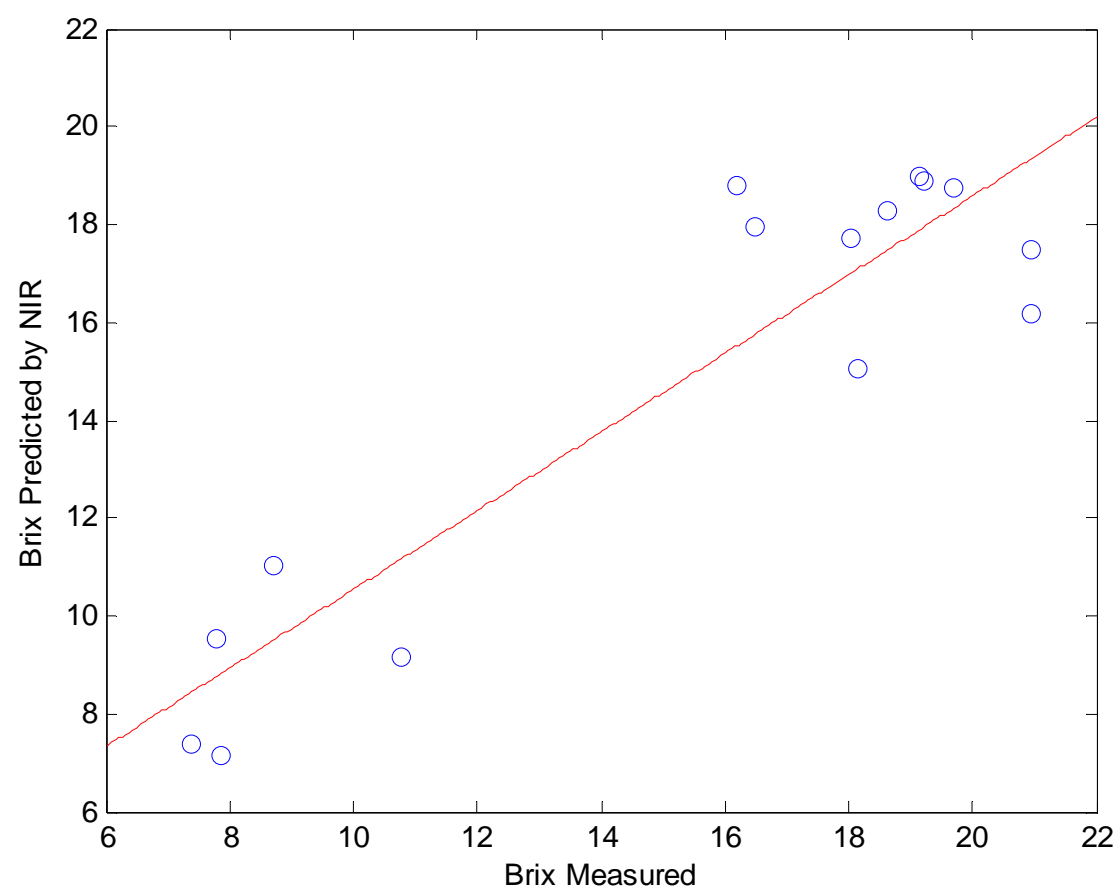

Fig. 3 Correlation between sugar content obtained through refractometer and sugar content predicted by spectral data through the PSLR method from the data extract from the husk.

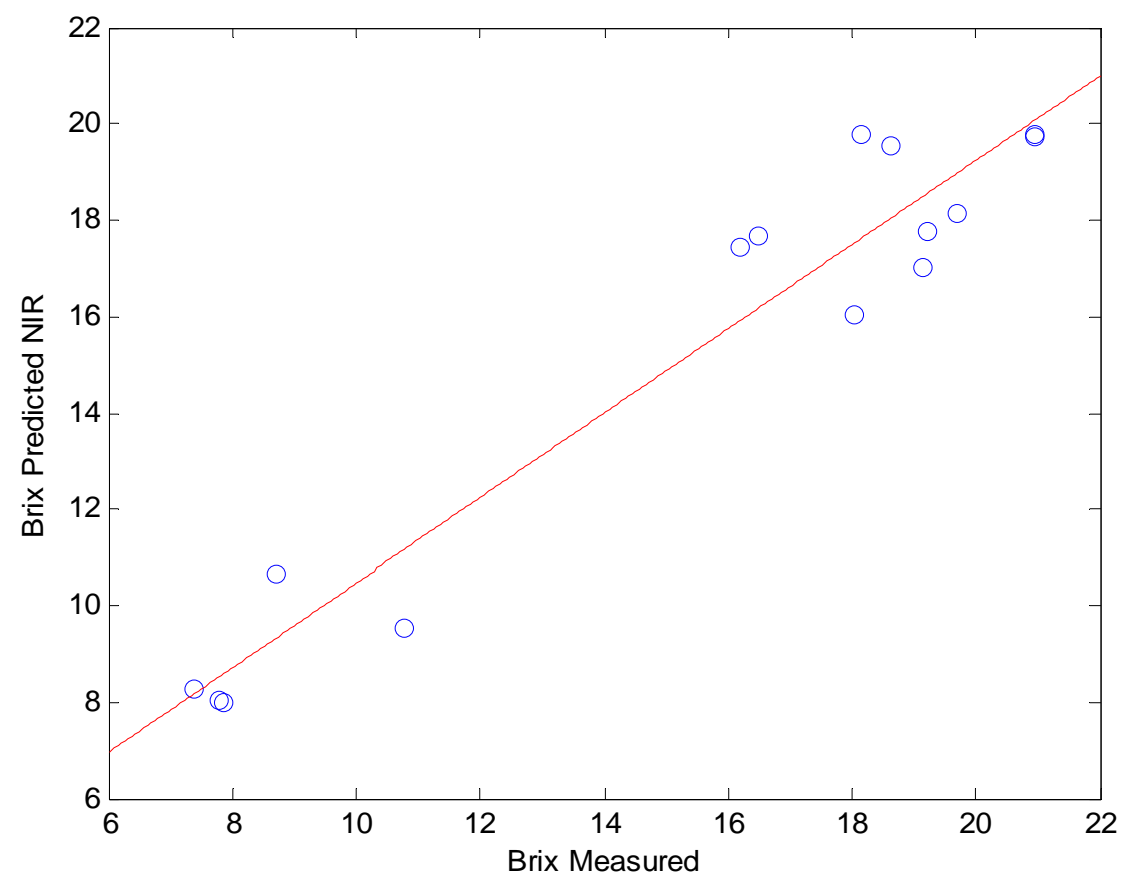

Fig. 4 Correlation between sugar content obtained through refractometer and the sugar content predicted by spectral data obtained from the internal part through the PSLR method.

Fig. 3 presents a correlation between the sugar content measured in laboratory (refractometer) and the value predicted by the model. The equation of the line is $y=$ $0.8039 x+2.5041\left(R^{2}=0.8378\right)$, where $y$ corresponds to the predicted Brix by NIR data extracted from the husk and $x$ corresponds to the measured Brix. The coefficient of determination indicates that $83.78 \%$ of predicted Brix can be explained by the proposed 
model. Sverzut et al. [10], working with the analysis of pol, fiber, sugar and moisture content in sugar cane samples, obtained good correlations with NIR spectroscopy and traditional methods.

The equation of the line from Fig. 4 is $y=0.8779 x$ $+1.699\left(R^{2}=0.9287\right)$, where $y$ corresponds to the predicted Brix by NIR data extracted from the internal part and $x$ corresponds to the measured Brix. It can be noted a high determination coefficient $\left(R^{2}\right)$, indicating that the model can explain the real data with $92.87 \%$ of adjustment. In works to determine the quality of sugar cane by means of near infra red spectroscopy, $R^{2}$ $=0.99$ between the Brix values and the values obtained by the model was obtained [11].

\section{Conclusions}

It was possible to differentiate the water contents of the sugar cane samples both externally lit (husk) and internally lit (cut) by Biospeckle analysis through the calculation of MI.

For the Biospeckle data and water content of the samples, it was possible to distinguish samples from the apex of the plant when the data were obtained from the sugarcane husk.

It was possible to obtain a model to predict the sugar content values of the samples from the sugar cane husk data, being a measurement of the external part, with no need for cutting or destroying the samples.

Thus, it is possible to evaluate the water content and the sugar content in the samples combining Biospeckle and NIR spectroscopy, which are non-invasive and non-destructive methods.

\section{References}

[1] Lemões, J. S., Oliveira, B. A., Farias, S. P., and Anjos-Silva, S. D. 1960. "Construction of the Calibration Curve for Prediction of Reducing Sugars and Total Sugar
Reducers in Sugarcane by Near Infrared Spectroscopy.” Empresa Brasileira de Pesquisa Agropecuária. Accessed September 27, 2015. http://ainfo.cnptia.embrapa.br/digital/bitstream/item/7529 0/1/075.pdf. (in Portuguese)

[2] Drennen, J. K., Kraemer, E. G., and Lodder, R. A. 1991. "Advances and Perspectives in Near-Infrared Spectrophotometry.” Crit. Rev. Anal. Chem. 22 (6): 443-75.

[3] Nawi, N. M., Chen, G. N., Jensen, T., and Baillie, C. 2012. "Prediction of Sugar Content along the Height of Sugarcane Internodes Using Vis/NIR.” University of Southern Queensland. Accessed January 21, 2016. https://eprints.usq.edu.au/21603/2/MatNawi_Chen_Jense n_Baillie_CIGR_2012b_AV.pdf.

[4] Ferraz, M. N. 2015. "Use of Spectrometry to Investigate the Quality of Sugarcane in the Field.” Accessed November 25, 2015. http://www.teses.usp.br/teses/disponiveis/11/11152/tde-1 6112015-145255/. (in Portuguese)

[5] Kurenda, A., Adamiak, A., and Zdunek, A. 2012. "Temperature Effect on Apple Biospeckle Activity Evaluated with Different Indices.” Postharvest Biol. Technol. 67: 118-23.

[6] Rabelo, G. F., Enes, A. M., Braga Junior, R. A., and Dal Fabbro, I. M. 2011. "Frequency Response of Biospeckle Laser Images of Bean Seeds Contaminated by Fungi.” Biosyst. Eng. 110 (3): 297-301.

[7] Zdunek, A., Adamiak, A., Pieczywek, P. M., and Kurenda, A. 2014. "The Biospeckle Method for the Investigation of Agricultural Crops: A Review.” Opt. Lasers Eng. 52: 276-85.

[8] Rabal, H. J., and Braga, R. A., eds. 2009. Dynamic Laser Speckle and Applications. Boca Raton: CRC Press.

[9] Ferreira, M. M. C. 2015. Chemometrics: Concepts, Methods and Applications. Campinas, SP: Unicamp. (in Portuguese)

[10] Sverzut, C. B., Verma, L. R., and French, A. D. 1987. "Sugarcane Analysis Using Near Infrared Spectroscopy." Transactions of the ASAE 30 (1): 255-8.

[11] Valderrama, P., Braga, J. W. B., and Poppi, R. J. 2007. "Validation of Multivariate Calibration Models in the Determination of Sugar Cane Quality Parameters by Near Infrared Spectroscopy.” J. Braz. Chem. Soc. 18 (2): 259-66. 